\title{
Formally Real Fields
}

\author{
Christoph Schwarzweller \\ Institute of Informatics \\ Faculty of Mathematics, Physics and Informatics \\ University of Gdańsk \\ Wita Stwosza 57, 80-308 Gdańsk, Poland
}

\begin{abstract}
Summary. We extend the algebraic theory of ordered fields [7, 6] in Mizar 1, 2, 3, : we show that every preordering can be extended into an ordering, i.e. that formally real and ordered fields coincide. We further prove some characterizations of formally real fields, in particular the one by Artin and Schreier using sums of squares [4. In the second part of the article we define absolute values and the square root function [5].
\end{abstract}

MSC: 12J15 03B35

Keywords: formally real fields; ordered fields; abstract value; square roots MML identifier: REALALG2, version: 8.1.06 5.45.1311

\section{Preliminaries}

Let $X, Y$ be non empty sets. Let us observe that there exists a function which is non empty, $X$-defined, and $Y$-valued and the carrier of $\mathbb{F}_{\mathbb{Q}}$ is rationalmembered.

Now we state the propositions:

(1) Let us consider a right zeroed, non empty additive loop structure $L$, and subsets $S, T$ of $L$. If $0_{L} \in T$, then $S \subseteq S+T$.

(2) Let us consider a right unital, non empty multiplicative loop structure $L$, and subsets $S, T$ of $L$. If $1_{L} \in T$, then $S \subseteq S \cdot T$.

(3) Let us consider an add-associative, right zeroed, right complementable, right distributive, non empty double loop structure $L$, and a subset $S$ of $L$. If $0_{L} \in S$, then for every element $a$ of $L, S \subseteq S+a \cdot S$. The theorem is a consequence of (1). 
(4) Let us consider an add-associative, right zeroed, right complementable, right unital, right distributive, non empty double loop structure $L$, and a subset $S$ of $L$. If $0_{L}, 1_{L} \in S$, then for every element $a$ of $L, a \in S+a \cdot S$.

(5) Let us consider an add-associative, right zeroed, right complementable, Abelian, left distributive, non empty double loop structure $R$, elements $a, b$ of $R$, and an integer $i$. Then $i \star(a \cdot b)=(i \star a) \cdot b$.

Proof: Define $\mathcal{P}$ [integer] $\equiv \$_{1} \star(a \cdot b)=\left(\$_{1} \star a\right) \cdot b . \mathcal{P}[0]$ by [11, (59)]. For every integer $u$ such that $\mathcal{P}[u]$ holds $\mathcal{P}[u-1]$ and $\mathcal{P}[u+1]$. For every integer $i, \mathcal{P}[i]$.

(6) Let us consider an add-associative, right zeroed, right complementable, Abelian, left distributive, non empty double loop structure $R$, an element $a$ of $R$, and an integer $i$. Then $i \star(-a)=-i \star a$.

Proof: Define $\mathcal{P}$ [integer] $\equiv \$_{1} \star(-a)=-\$_{1} \star a . \mathcal{P}[0]$. For every integer $u$ such that $\mathcal{P}[u]$ holds $\mathcal{P}[u-1]$ and $\mathcal{P}[u+1]$. For every integer $i, \mathcal{P}[i]$.

Let $R$ be a ring and $a$ be an element of $R$. Let us consider a commutative ring $R$ and elements $a, b$ of $R$. Now we state the propositions:

(7) $(a+b)^{2}=a^{2}+2 \star a \cdot b+b^{2}$. The theorem is a consequence of (43).

(8) $(a-b)^{2}=a^{2}-2 \star a \cdot b+b^{2}$. The theorem is a consequence of (7), (43), and $(6)$.

(9) $(a+b) \cdot(a-b)=a^{2}-b^{2}$.

(10) Let us consider an integral domain $R$, and elements $a, b$ of $R$. Then $a^{2}=b^{2}$ if and only if $a=b$ or $a=-b$. The theorem is a consequence of (9).

Let us consider a field $F$ and a non zero element $a$ of $F$. Now we state the propositions:

(11) $(-a)^{-1}=-a^{-1}$.

(12) $\left(-a^{-1}\right)^{-1}=-a$.

(13) $-(-a)^{-1}=a^{-1}$. The theorem is a consequence of (11).

(14) Let us consider a field $F$, an element $a$ of $F$, and a non zero element $b$ of $F$. Then $\left(\frac{a}{b}\right)^{2}=\frac{a^{2}}{b^{2}}$.

(15) Let us consider a field $F$. Suppose $\operatorname{char}(F) \neq 2$. Let us consider an element $a$ of $F$. Then $\left(\frac{a+1_{F}}{2 \star 1_{F}}\right)^{2}-\left(\frac{a-1_{F}}{2 \star 1_{F}}\right)^{2}=a$. The theorem is a consequence of (14), (7), (8), and (43).

Let us note that every non degenerated ring which is preordered has also characteristic 0 . Let us consider a preordered $\operatorname{ring} R$ and a preordering $P$ of $R$. Now we state the propositions:

(16) $(-P) \cdot P=P \cdot(-P)$.

(17) (i) $-P+-P \subseteq-P$, and 
(ii) $(-P) \cdot(-P) \subseteq P$.

(18)

(i) $(-P) \cdot P \subseteq-P$, and

(ii) $P \cdot(-P) \subseteq-P$.

The theorem is a consequence of (17) and (16).

(19) Let us consider a preordered ring $R$, a preordering $P$ of $R$, and a natural number $n$. Then $n \star 1_{R} \in P$.

Proof: Define $\mathcal{P}$ [natural number] $\equiv \$_{1} \star 1_{R} \in P$. For every natural number $k, \mathcal{P}[k]$.

One can verify that every preordering of $\mathbb{Z}^{\mathrm{R}}$ is spanning and every preordering of $\mathbb{F}_{\mathbb{Q}}$ is spanning and every preordering of $\mathbb{R}_{\mathrm{F}}$ is spanning.

(20) Let us consider a preordering $P$ of $\mathbb{Z}^{\mathrm{R}}$. Then $P=\operatorname{Positives}\left(\mathbb{Z}^{\mathrm{R}}\right)$.

(21) Let us consider a preordering $P$ of $\mathbb{F}_{\mathbb{Q}}$. Then $P=\operatorname{Positives}\left(\mathbb{F}_{\mathbb{Q}}\right)$.

(22) Let us consider a preordering $P$ of $\mathbb{R}_{\mathrm{F}}$. Then $P=\operatorname{Positives}\left(\mathbb{R}_{\mathrm{F}}\right)$.

\section{More on Ring Characteristic}

Now we state the propositions:

(23) Let us consider a $\operatorname{ring} R$. Then $\operatorname{char}(R)=1$ if and only if $R$ is degenerated.

(24) Let us consider a non degenerated ring $R$. Then $\operatorname{char}(R)=2$ if and only if $2 \star 1_{R}=0_{R}$.

(25) Let us consider an integral domain $R$. Then $\operatorname{char}(R)=0$ if and only if for every non zero element $a$ of $R$ and for every non zero natural number $n, n \star a \neq 0_{R}$. The theorem is a consequence of (43).

(26) Let us consider an integral domain $R$ with characteristic 0 , and an element $a$ of $R$. Then $-a=a$ if and only if $a=0_{R}$. The theorem is a consequence of (25).

\section{Maximal Preorderings}

Let $R$ be a preordered ring and $P$ be a preordering of $R$. We say that $P$ is maximal if and only if

(Def. 1) for every preordering $Q$ of $R$ such that $P \subseteq Q$ holds $P=Q$.

Now we state the propositions:

(27) Let us consider a preordered field $F$, a preordering $P$ of $F$, and an element $a$ of $F$. If $-a \notin P$, then $P+(a \cdot P)$ is a preordering of $F$.

Proof: Set $S=P+(a \cdot P) . S+S \subseteq S . S \cdot S \subseteq S$ by [8, (8), (10)], [10, (3)], [9, (23)]. $P \subseteq S$. 
(28) Let us consider a preordered field $F$, and a preordering $P$ of $F$. Then $P$ is maximal if and only if $P$ is a positive cone. The theorem is a consequence of (36), (3), and (4).

Let $F$ be a preordered field. Note that every preordering of $F$ which is spanning is also maximal and every preordering of $F$ which is maximal is also spanning. Now we state the proposition:

(29) Let us consider a preordered field $F$, and a preordering $P$ of $F$. Then there exists a preordering $Q$ of $F$ such that

(i) $P \subseteq Q$, and

(ii) $Q$ is maximal.

Let us note that every preordered field is ordered. Let us consider a preordered field $F$ and a preordering $P$ of $F$. Now we state the propositions:

(30) $P$ is maximal if and only if $P$ is an ordering of $F$.

(31) There exists an ordering $O$ of $F$ such that $P \subseteq O$. The theorem is a consequence of (29).

Let $R$ be an ordered ring and $P$ be a preordering of $R$. The functor $\bigcap_{R} P$ yielding a subset of $R$ is defined by the term

(Def. 2) $\quad\{x$, where $x$ is an element of $R$ : for every ordering $O$ of $R$ such that $P \subseteq O$ holds $x \in O\}$.

One can verify that $\bigcap_{R} P$ is non empty and $\bigcap_{R} P$ is closed under addition and closed under multiplication and has all squares.

Let $F$ be an ordered field and $P$ be a preordering of $F$. One can verify that $\bigcap_{F} P$ is negative-disjoint. Let us consider an ordered field $F$ and a preordering $P$ of $F$. Now we state the propositions:

(32) $\bigcap_{F} P$ is a preordering of $F$.

(33) $\bigcap_{F} P=P$.

\section{Formally Real Fields}

Let $R$ be a ring. We say that $R$ is formally real if and only if (Def. 3) $-1_{R} \notin \mathrm{QS}(R)$.

Let us consider a field $F$. Now we state the propositions:

(34) If $\operatorname{char}(F) \neq 2$, then $F$ is formally real iff $\mathrm{QS}(F)$ is a prepositive cone.

(35) If $\operatorname{char}(F) \neq 2$, then $F$ is formally real iff there exists a subset $P$ of $F$ such that $P$ is a prepositive cone.

(36) If $\operatorname{char}(F) \neq 2$, then $F$ is formally real iff there exists a subset $P$ of $F$ such that $P$ is a positive cone. 
(37) If $\operatorname{char}(F) \neq 2$, then $F$ is formally real iff $\mathrm{QS}(F) \neq$ the carrier of $F$.

Observe that every field which is formally real is also ordered and every field which is ordered is also formally real and every non degenerated ring which is preordered is also formally real and there exists a field which is formally real.

Let $F$ be a formally real field. Note that $\mathrm{QS}(F)$ is negative-disjoint.

Now we state the propositions:

(38) Let us consider a formally real field $F$. Then $\mathrm{QS}(F)$ is a preordering of $F$.

(39) Let us consider a formally real field $F$, and an element $a$ of $F$. Then for every ordering $O$ of $F, a \in O$ if and only if $a \in \operatorname{QS}(F)$.

(40) Let us consider an element $r$ of $\mathbb{F}_{\mathbb{Q}}$. If $0 \leqslant r$, then $r$ is a sum of squares.

Let $R$ be a zero structure and $f$ be a (the carrier of $R$ )-valued function. We say that $f$ is trivial if and only if

(Def. 4) for every object $i$ such that $i \in \operatorname{dom} f$ holds $f(i)=0_{R}$.

Let $R$ be a ring and $f$ be a non empty finite sequence of elements of $R$. We say that $f$ is quadratic if and only if

(Def. 5) for every element $i$ of $\operatorname{dom} f, f(i)$ is a square.

Let $R$ be a non degenerated ring. Observe that $\left\langle 1_{R}\right\rangle$ is quadratic and non trivial as a non empty finite sequence of elements of $R$ and there exists a non empty finite sequence of elements of $R$ which is quadratic and non trivial.

Now we state the proposition:

(41) Let us consider a field $F$. Then $F$ is formally real if and only if for every quadratic, non empty finite sequence $f$ of elements of $F$ such that $\sum f=0_{F}$ holds $f$ is trivial.

Note that every formally real field is non algebraic closed.

\section{Order Relations and Strict Order Relations Revisited}

Now we state the propositions:

(42) Let us consider a preordered ring $R$, a preordering $P$ of $R$, and elements $a, b$ of $R$. Then $a \leqslant P b$ if and only if $-b \leqslant P-a$.

(43) Let us consider a preordered ring $R$, a preordering $P$ of $R$, and an element $a$ of $R$. Then $a \leqslant P$.

(44) Let us consider a preordered ring $R$, a preordering $P$ of $R$, and elements $a, b$ of $R$. If $a \leqslant_{P} b$ and $b \leqslant_{P} a$, then $a=b$.

Let us consider a preordered ring $R$, a preordering $P$ of $R$, and elements $a$, $b, c$ of $R$. Now we state the propositions: 
(45) If $a \leqslant_{P} b$ and $b \leqslant_{P} c$, then $a \leqslant P c$.

(46) If $a \leqslant_{P} b$, then $a+c \leqslant_{P} b+c$.

(47) If $a \leqslant_{P} b$ and $0_{R} \leqslant P c$, then $a \cdot c \leqslant_{P} b \cdot c$.

(48) If $a \leqslant_{P} b$ and $c \leqslant_{P} 0_{R}$, then $b \cdot c \leqslant_{P} a \cdot c$. The theorem is a consequence of (47) and (42).

(49) Let us consider an ordered ring $R$, an ordering $O$ of $R$, and elements $a$, $b$ of $R$. Then

(i) $a \leqslant 0 b$, or

(ii) $b \leqslant O a$.

Let us consider a preordered field $F$, a preordering $P$ of $F$, and non zero elements $a, b$ of $F$. Now we state the propositions:

(50) If $0_{F} \leqslant_{P} a$ and $0_{F} \leqslant_{P} b$, then $a \leqslant_{P} b$ iff $b^{-1} \leqslant_{P} a^{-1}$. The theorem is a consequence of $(47)$.

(51) If $a \leqslant_{P} 0_{F}$ and $b \leqslant_{P} 0_{F}$, then $a \leqslant_{P} b$ iff $b^{-1} \leqslant_{P} a^{-1}$. The theorem is a consequence of (13) and (48).

Let $R$ be a preordered ring, $P$ be a preordering of $R$, and $a, b$ be elements of $R$. We say that $a<_{P} b$ if and only if

(Def. 6) $\quad a \leqslant_{P} b$ and $a \neq b$.

Now we state the propositions:

(52) Let us consider a preordered, non degenerated ring $R$, and a preordering $P$ of $R$. Then

(i) $0_{R}<P 1_{R}$, and

(ii) $-1_{R}<_{P} 0_{R}$.

(53) Let us consider a preordered ring $R$, a preordering $P$ of $R$, and elements $a, b$ of $R$. Then $a<_{P} b$ if and only if $-b<_{P}-a$.

(54) Let us consider an ordered ring $R$, an ordering $O$ of $R$, and elements $a$, $b$ of $R$. Then

(i) $a<_{O} b$, or

(ii) $b<_{O} a$, or

(iii) $a=b$.

Let us consider a preordered ring $R$, a preordering $P$ of $R$, and elements $a$, $b, c$ of $R$. Now we state the propositions:

(55) If $a<_{P} b$ and $b \leqslant_{P} c$, then $a<_{P} c$.

(56) If $a \leqslant_{P} b$ and $b<_{P} c$, then $a<_{P} c$.

(57) If $a<_{P} b$, then $a+c<_{P} b+c$. 
Let us consider a preordered integral domain $R$, a preordering $P$ of $R$, and elements $a, b, c$ of $R$. Now we state the propositions:

(58) If $a<_{P} b$ and $0_{R}<_{P} c$, then $a \cdot c<_{P} b \cdot c$.

(59) If $a<_{P} b$ and $c<_{P} 0_{R}$, then $b \cdot c<_{P} a \cdot c$. The theorem is a consequence of (42) and (47).

Let us consider a preordered field $F$, a preordering $P$ of $F$, and non zero elements $a, b$ of $F$. Now we state the propositions:

(60) If $0_{F} \leqslant_{P} a$ and $0_{F} \leqslant_{P} b$, then $a<_{P} b$ iff $b^{-1}<_{P} a^{-1}$. The theorem is a consequence of $(50)$.

(61) If $a \leqslant_{P} 0_{F}$ and $b \leqslant_{P} 0_{F}$, then $a<_{P} b$ iff $b^{-1}<_{P} a^{-1}$. The theorem is a consequence of $(51)$.

Let $R$ be a preordered ring, $P$ be a preordering of $R$, and $a$ be an element of $R$. We say that $a$ is $P$-ordered if and only if

(Def. 7) $a \in P \cup-P$.

We say that $a$ is $P$-positive if and only if

(Def. 8) $a \in P \backslash\left\{0_{R}\right\}$.

We say that $a$ is $P$-negative if and only if

(Def. 9) $a \in-P \backslash\left\{0_{R}\right\}$.

Note that there exists an element of $R$ which is $P$-ordered and every element of $R$ which is $P$-positive is also $P$-ordered and every element of $R$ which is $P$ negative is also $P$-ordered.

Let $R$ be a preordered, non degenerated ring. One can check that there exists an element of $R$ which is $P$-positive and there exists an element of $R$ which is $P$-negative and there exists an element of $R$ which is non $P$-positive and there exists an element of $R$ which is non $P$-negative and every element of $R$ which is $P$-positive is also non zero and non $P$-negative and every element of $R$ which is $P$-negative is also non zero and non $P$-positive.

Let $a$ be a $P$-ordered element of $R$. One can verify that $-a$ is $P$-ordered.

Let $F$ be a field and $a$ be a non zero element of $F$. Let us note that $a^{-1}$ is non zero.

Let $F$ be a preordered field, $P$ be a preordering of $F$, and $a$ be a non zero, $P$-ordered element of $F$. Let us observe that $a^{-1}$ is $P$-ordered.

Let $R$ be an ordered, non degenerated ring and $O$ be an ordering of $R$. Note that every element of $R$ which is non zero and non $O$-positive is also $O$-negative and every element of $R$ which is non zero and non $O$-negative is also $O$-positive.

Let us consider a preordered ring $R$, a preordering $P$ of $R$, and an element $a$ of $R$. Now we state the propositions:

(62) $a$ is $P$-positive if and only if $0_{R}<_{P} a$. 
(63) $a$ is $P$-negative if and only if $a<_{P} 0_{R}$.

Let us consider a preordered ring $R$, a preordering $P$ of $R$, and a $P$-ordered element $a$ of $R$. Now we state the propositions:

(64) $a$ is not $P$-negative if and only if $0_{R} \leqslant P$. The theorem is a consequence of (43).

(65) $a$ is not $P$-positive if and only if $a \leqslant P 0_{R}$. The theorem is a consequence of (43).

\section{Absolute Values}

Let $R$ be a preordered ring, $P$ be a preordering of $R$, and $a$ be an element of $R$. The functor $|a|_{P}$ yielding an element of $R$ is defined by the term

(Def. 10) $\begin{cases}a, & \text { if } a \in P, \\ -a, & \text { if } a \in-P, \\ -1_{R}, & \text { otherwise. }\end{cases}$

Let $R$ be an ordered ring and $O$ be an ordering of $R$. One can verify that the functor $|a|_{O}$ yields an element of $R$ and is defined by the term

(Def. 11) $\begin{cases}a, & \text { if } a \in O, \\ -a, & \text { otherwise. }\end{cases}$

Let us consider a preordered, non degenerated ring $R$, a preordering $P$ of $R$, and an element $a$ of $R$. Now we state the propositions:

(66) $0_{R} \leqslant P|a|_{P}$ if and only if $a$ is $P$-ordered. The theorem is a consequence of (55) and (52).

(67) $a$ is not $P$-ordered if and only if $|a|_{P}=-1_{R}$. The theorem is a consequence of (66).

(68) $|a|_{P}=0_{R}$ if and only if $a=0_{R}$. The theorem is a consequence of (67).

Let us consider a preordered integral domain $R$, a preordering $P$ of $R$, and an element $a$ of $R$. Now we state the propositions:

(69) $|a|_{P}=a$ if and only if $0_{R} \leqslant P a$. The theorem is a consequence of (26) and (43).

(70) $|a|_{P}=-a$ if and only if $a \leqslant{ }_{P} 0_{R}$.

(71) Let us consider a preordered ring $R$, a preordering $P$ of $R$, and an element $a$ of $R$. Then $|a|_{P}=|-a|_{P}$.

(72) Let us consider a preordered, non degenerated ring $R$, a preordering $P$ of $R$, and an element $a$ of $R$. Then $-|a|_{P} \leqslant_{P} a$ and $a \leqslant_{P}|a|_{P}$ if and only if $a$ is $P$-ordered. The theorem is a consequence of (45), (52), (56), (44), and (66). 
(73) Let us consider a preordered field $F$, a preordering $P$ of $F$, and a non zero, $P$-ordered element $a$ of $F$. Then $\left|a^{-1}\right|_{P}=\left(|a|_{P}\right)^{-1}$.

Proof: $\left|a^{-1}\right|_{P} \cdot|a|_{P}=\mathbf{1}_{F}$.

(74) Let us consider a preordered ring $R$, a preordering $P$ of $R$, and elements $a, b$ of $R$. Then $|(a-b)|_{P}=|(b-a)|_{P}$.

(75) Let us consider a preordered, non degenerated ring $R$, a preordering $P$ of $R$, and an element $a$ of $R$. Then $-|a|_{P} \leqslant_{P} a$ and $a \leqslant_{P}|a|_{P}$ if and only if $a$ is $P$-ordered. The theorem is a consequence of (67), (52), (44), (45), and (43).

(76) Let us consider a preordered, non degenerated ring $R$, a preordering $P$ of $R$, and $P$-ordered elements $a, b$ of $R$. Then $|(a \cdot b)|_{P}=\left(|a|_{P}\right) \cdot\left(|b|_{P}\right)$. The theorem is a consequence of (18) and (17).

(77) Let us consider a preordered field $F$, a preordering $P$ of $F$, a non zero, $P$ ordered element $a$ of $F$, and a $P$-ordered element $b$ of $F$. Then $\left|b \cdot\left(a^{-1}\right)\right|_{P}=$ $|b|_{P} \cdot\left(|a|_{P}\right)^{-1}$. The theorem is a consequence of (76) and (73).

(78) Let us consider a preordered integral domain $R$, a preordering $P$ of $R$, a $P$-ordered element $a$ of $R$, and a $P$-ordered, non $P$-negative element $p$ of $R$. Then $|a|_{P} \leqslant_{P} p$ if and only if $-p \leqslant_{P} a$ and $a \leqslant_{P} p$. The theorem is a consequence of (75), (45), (42), (69), and (70).

(79) Let us consider a preordered integral domain $R$, a preordering $P$ of $R$, and $P$-ordered elements $a, b$ of $R$. Then $|(a+b)|_{P} \leqslant P|a|_{P}+|b|_{P}$. The theorem is a consequence of (66), (46), (45), (52), (53), (44), (75), and (78).

\section{Squares and Square Roots}

Let $R$ be a ring and $a$ be square element of $R$.

A square root of $a$ is an element of $R$ defined by

(Def. 12) $i t^{2}=a$.

Let $R$ be a non degenerated ring. Observe that there exists an element of $R$ which is non zero and square.

Let us consider an ordered integral domain $R$, an ordering $O$ of $R$, and non $O$-negative elements $a, b$ of $R$. Now we state the propositions:

(80) $a \leqslant_{O} b$ if and only if $a^{2} \leqslant_{O} b^{2}$. The theorem is a consequence of (64), (47), (45), (18), and (9).

(81) $a<_{O} b$ if and only if $a^{2}<_{O} b^{2}$. The theorem is a consequence of (64) and (80). 
(82) Let us consider a preordered integral domain $R$, a preordering $P$ of $R$, and a $P$-ordered element $a$ of $R$. Then $\left(|a|_{P}\right)^{2}=a^{2}$. The theorem is a consequence of (69) and (70).

(83) Let us consider a preordered ring $R$, a preordering $P$ of $R$, and an element $a$ of $R$. If $a \in-P \backslash\left\{0_{R}\right\}$, then $a$ is not square.

(84) Let us consider a preordered ring $R$, and a preordering $P$ of $R$. Then $(-P) \cap \mathrm{SQ}(R)=\left\{0_{R}\right\}$. The theorem is a consequence of (83).

(85) Let us consider a preordered integral domain $R$, a preordering $P$ of $R$, square element $a$ of $R$, and square roots $b_{1}, b_{2}$ of $a$. If $0_{R} \leqslant_{P} b_{1}$ and $0_{R} \leqslant P b_{2}$, then $b_{1}=b_{2}$.

Let $R$ be a preordered ring and $P$ be a preordering of $R$. Let us observe that every element of $R$ which is $P$-negative is also non square and every element of $R$ which is non $P$-positive and square is also zero.

Let $R$ be an ordered integral domain, $O$ be an ordering of $R$, and $a$ be square element of $R$. One can check that there exists a square root of $a$ which is non $O$-negative.

Let $a$ be a non zero, a square element of $R$. Let us observe that there exists a square root of $a$ which is $O$-positive and there exists a square root of $a$ which is $O$-negative.

Let $a$ be square element of $R$. The functor $\sqrt{a} O$ yielding a non $O$-negative square root of $a$ is defined by

(Def. 13) $i t^{2}=a$.

Now we state the proposition:

(86) Let us consider an ordered integral domain $R$, an ordering $O$ of $R$, square element $a$ of $R$, and an element $b$ of $R$. Then $b$ is a square root of $a$ if and only if $b=\sqrt{a}_{O}$ or $b=-\sqrt{a}_{O}$. The theorem is a consequence of (10).

Let $R$ be an ordered integral domain, $O$ be an ordering of $R$, and $a$ be a non zero, a square element of $R$. One can check that $\sqrt{a}_{O}$ is non zero.

\section{REFERENCES}

[1] Grzegorz Bancerek, Czesław Byliński, Adam Grabowski, Artur Korniłowicz, Roman Matuszewski, Adam Naumowicz, Karol Pąk, and Josef Urban. Mizar: State-of-the-art and beyond. In Manfred Kerber, Jacques Carette, Cezary Kaliszyk, Florian Rabe, and Volker Sorge, editors, Intelligent Computer Mathematics, volume 9150 of Lecture Notes in Computer Science, pages 261-279. Springer International Publishing, 2015. ISBN 978-3319-20614-1. doi 10.1007/978-3-319-20615-8_17.

[2] Adam Grabowski, Artur Korniłowicz, and Adam Naumowicz. Four decades of Mizar. Journal of Automated Reasoning, 55(3):191-198, 2015. doi $10.1007 / \mathrm{s} 10817-015-9345-1$

[3] Adam Grabowski, Artur Korniłowicz, and Christoph Schwarzweller. On algebraic hierarchies in mathematical repository of Mizar. In M. Ganzha, L. Maciaszek, and M. Paprzycki, editors, Proceedings of the 2016 Federated Conference on Computer Science and Infor- 
mation Systems (FedCSIS), volume 8 of Annals of Computer Science and Information Systems, pages 363-371, 2016. doi $10.15439 / 2016$ F520.

[4] Nathan Jacobson. Lecture Notes in Abstract Algebra, III. Theory of Fields and Galois Theory. Springer-Verlag, 1964.

[5] Manfred Knebusch and Claus Scheiderer. Einführung in die reelle Algebra. Vieweg-Verlag, 1989.

[6] Alexander Prestel. Lectures on Formally Real Fields. Springer-Verlag, 1984.

[7] Knut Radbruch. Geordnete Körper. Lecture Notes, University of Kaiserslautern, Germany, 1991.

[8] Christoph Schwarzweller. The binomial theorem for algebraic structures. Formalized Mathematics, 9(3):559-564, 2001.

[9] Christoph Schwarzweller. Ordered rings and fields. Formalized Mathematics, 25(1):63-72, 2017. doi $10.1515 /$ forma-2017-0006.

[10] Christoph Schwarzweller. On roots of polynomials and algebraically closed fields. Formalized Mathematics, 25(3):185-195, 2017. doi:10.1515/forma-2017-0018.

[11] Christoph Schwarzweller and Artur Korniłowicz. Characteristic of rings. Prime fields. Formalized Mathematics, 23(4):333-349, 2015. doi 10.1515/forma-2015-0027.

Received November 29, 2017

The English version of this volume of Formalized Mathematics was financed under agreement 548/P-DUN/2016 with the funds from the Polish Minister of Science and Higher Education for the dissemination of science. 